\title{
Luminescent properties of a water-soluble conjugated polymer incorporating graphene oxide quantum dots
}

\author{
Penglei Li, ${ }^{1}$ Francesco Di Stasio, ${ }^{1}$ Goki Eda, ${ }^{2}$ Oliver Fenwick, ${ }^{1}$ Shane $O$. \\ McDonnell, ${ }^{3}$ Harry L. Anderson, ${ }^{3}$ Manish Chhowalla, ${ }^{4}$ and Franco Cacialli ${ }^{*}$ \\ ${ }^{1}$ London Centre for Nanotechnology and Department of Physics and Astronomy, \\ University College London, Gower Street, London WC1E 6BT, UK. \\ ${ }^{2}$ Department of Physics, National University of Singapore, 2 Science Drive 3, \\ Singapore 117542. \\ ${ }^{3}$ Department of Chemistry, University of Oxford, Chemistry Research Laboratory, \\ Mansfield Road, Oxford OX1 3TA, UK. \\ ${ }^{4}$ Materials Science and Engineering, Rutgers University, 607 Taylor Road, \\ Piscataway, New Jersey 08854, USA
}

\begin{abstract}
We report the incorporation of graphene oxide quantum dots (GOQDs) into films, diluted solutions and light-emitting diodes (LEDs) of a water-soluble derivative of poly(p-phenylene vinylene), or PDV.Li, to investigate their impact on the light-emission properties of this model conjugated polymer. Despite the wellknown ability of graphene and graphene oxide to quench the photoluminescence of nearby emitters, we find that addition of GOQDs to diluted solutions of PDV.Li does not affect significantly the photoluminescence (PL) dynamics of PDV.Li, bringing about only a modest quenching of the PL. On the other hand, loading with GOQD led to substantial decrease in the turn-on voltage of LEDs based on GOQD-PDV.Li composites. This effect is either due to improved morphology of the host polymer and a resulting increase in the charge mobility or is due to enhanced injection via GOQDs near the electrodes.
\end{abstract}

*Corresponding author: f.cacialli@ucl.ac.uk 
Graphene and its chemical derivatives have been attracting huge academic and industrial interest due to their potential in a wide range of applications. ${ }^{[1]}$ Graphene oxide $(\mathrm{GO})^{[2]}$ is a water-soluble chemical derivative of graphene wellknown for its scalable synthesis and solution-processability. ${ }^{[2 b, 2 c, 2 f, 3]}$ GO can be viewed as constituting of a matrix of hexagonally packed $\mathrm{sp}^{2}$ and $\mathrm{sp}^{3}$ hybridised carbon atoms with covalently attached oxygen functional groups. Unlike graphene, which is semimetallic, GO is a photoluminescent insulator with luminescent localized states. ${ }^{[2 \mathrm{~b}, 2 \mathrm{2f}]}$ Oxidative cutting of GO results in formation of so-called GO quantum dots (GOQDs) with typical lateral dimensions of $10 \sim 15 \mathrm{~nm}{ }^{[4]}$ While GOQDs are chemically similar to GO, they exhibit higher water-solubility and typically distinct photoluminescence (PL) characteristics, which occur in the visible to the near-IR range. ${ }^{[3]}$ These properties of GO and GOQDs make them potentially useful for optoelectronics ${ }^{[2 \mathrm{e}]}$, drug-delivery ${ }^{[2 \mathrm{~g}]}$ and biomedical sensing. ${ }^{[5]}$

Incorporation of GO sheets and GOQDs into polymeric materials enables exploration of new functionalities arising from the interaction between the guest and the host material. For example, use of reduced GO (rGO) as an electron-acceptor in bulk heterojunction solar cells has been recently demonstrated. ${ }^{[6]}$ However, asprepared GO is essentially hydrophilic, and cannot be used directly in blends with most conjugated polymers, which are commonly soluble in organic solvents. Appropriate functionalization of $\mathrm{GO}$ is therefore needed to achieve their solubilisation in the same organic solvent as the conjugated polymers. ${ }^{[2 \mathrm{~b}, 2 \mathrm{~g}, 7]}$ The alternative approach that we explore here is to utilize polymers that are soluble in water or polar solvents, such as the precursor to $\operatorname{poly}\left(p\right.$-phenylene vinylene) $(\mathrm{PPV})^{[8]}$ or derivatives of poly(4,4-diphenylene vinylene) carrying lithium-sulphonated water-solubilising chains (PDV.Li, for the chemical structure see supporting information, Fig. S1). ${ }^{[9]}$ The latter polyelectrolytes, for example, have been previously studied as model systems to investigate the potential to prevent aggregation and subsequent PL quenching by "rotaxination", i.e. by threading of the conjugated backbones into insulating cyclodextrin rings that act as spacers. ${ }^{[9 \mathrm{~b}, 9 \mathrm{c}]} \mathrm{In}$ previous work we also found that polyethylene oxide (PEO $)^{[10]}$ wraps around the conjugated polyanionic backbones (as it also does with DNA) thereby mitigating aggregation quenching, and that a similar effect is also observed upon substitution of the $\mathrm{Li}$ counter-cations with cationic species of increasing sizes. ${ }^{[11]} \mathrm{We}$ considered that the hydrophilic groups of GOQDs might also closely interact with the polar 
groups of the conjugated polyelectrolytes, as in the case of PEO, and that this effect could also contribute to separate the polymer chains, thus suppressing aggregation and PL quenching. This is particularly interesting because while rotaxination is very effective at controlling the photophysics of the conjugated cores, and even enables ultra-broad gain bands ${ }^{[12]}$ and fabrication of white-emitting LEDs, ${ }^{[13]}$ wrapping of the backbones with cyclodextrin macrocycles or amylose linear chains ${ }^{[14]}$ does impair charge transport, with a concomitant and undesirable increase of the turn-on and operating voltages of light-emitting diodes (LEDs).

Here, we therefore explore the incorporation of GOQDs into films, diluted solutions and LEDs based on PDV.Li, to investigate their impact on the conjugated polymer optical (and electroluminescent) properties. While graphene and GO are well known to quench the PL from nearby molecules by efficient energy transfer, ${ }^{[15]}$ we find that addition of GOQDs to diluted solutions of PDV.Li does not affect significantly the PL dynamics of PDV.Li, bringing about only a modest quenching of the PL. Furthermore, we show that LEDs based on GOQD-PDV.Li composites exhibit significantly reduced turn-on voltage compared to the neat PDV.Li devices.

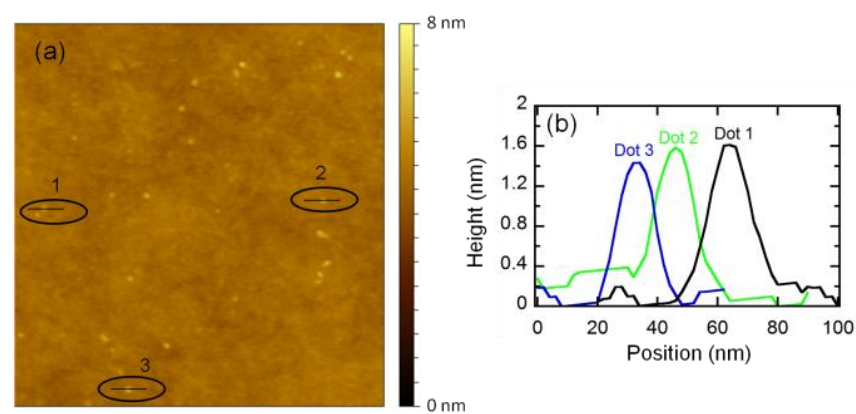

Figure 1 (a) Tapping-mode atomic force microscopy (AFM) images of GOQDs deposited on a $\mathrm{Si} / \mathrm{SiO}_{2}$ substrate. GOQDs were deposited from water solution with a concentration of $0.5 \mathrm{mg} / \mathrm{ml}$ by spin-coating. Following the deposition the sample was annealed at $150{ }^{\circ} \mathrm{C}$ in glove box for 1 hour. (b) Thickness profile of the selected three GOQDs shown in (a). These are representative of most GOQDs which are found to be characterized by a height of $1.4 \mathrm{~nm}$ and a lateral size of $10-15 \mathrm{~nm}$ at FWHM. No tip-deconvolution correction has been applied.

Starting our discussion with the morphological and structural properties, we report a tapping-mode atomic force microscopy (AFM) image of GOQDs on a $\mathrm{Si} / \mathrm{SiO}_{2}$ substrate in Fig. 1. An apparently uniform distribution of GOQDs is observed, with lateral sizes between 10 and $15 \mathrm{~nm}$ (full-width at half maximum, FWHM,), and thickness of $\sim 1.4 \mathrm{~nm}$. Such thicknesses correspond to $1-2$ layers of GO sheets in 
agreement with earlier work. Previously, nano-GO functionalised with polyethylene glycol (PEG), or "PEGylated nano-GO"[2g] has been reported as a composite in which the PEG chains help to break as-prepared GO sheets $(10$ - $300 \mathrm{~nm})$ into smaller flakes with average dimensions of $20 \mathrm{~nm}$.

Absorption and PL spectra of neat PDV.Li and GOQDs in solutions (0.05 $\mathrm{mg} / \mathrm{ml}$ ) are presented in Fig. 2a and b. Here we observe the typical absorption peak of GOQDs at $5.5 \mathrm{eV}(225 \mathrm{~nm})$, corresponding to the $\pi-\pi^{*}$ resonant transition. It is worth noting that unlike $\mathrm{GO}^{[3,16]}$ and graphene quantum dots (GQDs) ${ }^{[17]}$, the absorption peak at $\sim 4.13 \mathrm{eV}(300 \mathrm{~nm})$, which is attributed to $\mathrm{n}-\pi^{*}$ transition, is absent in our GOQDs. The PL spectrum of GOQDs peaks at $2.17 \mathrm{eV}(572 \mathrm{~nm})$, very close to the previously reported emission from nano-GO $(20 \mathrm{~nm})^{[2 \mathrm{~g}]}$ and GQDs (1$4 \mathrm{~nm}) .{ }^{[17 \mathrm{~b}]}$ Another emission component centred at around $2.81 \mathrm{eV}(440 \mathrm{~nm})$ is also evident from the emission spectrum. This peak has been previously observed for 5 $10 \mathrm{~nm}$ GQDs. ${ }^{[17 \mathrm{a}]-[18]}$ Such blue emission is ascribed to recombination of electronhole pairs generated within $\mathrm{sp}^{2}$ cluster domains ${ }^{[2 b, 3]}$ while the yellow-red emission $(\sim 572 \mathrm{~nm})$ may be associated with the disorder-induced localized states which lie within the $\pi-\pi^{*}$ gap, as recently discussed for GO. ${ }^{[19]}$

Figure 2c shows the PL spectra of PDV.Li:GOQDs blends with different weight ratios in aqueous solution (concentration of PDV.Li is kept constant at 0.05 $\mathrm{mg} / \mathrm{ml})$. PDV.Li shows a main PL peak at $2.48 \mathrm{eV}(500 \mathrm{~nm})$ with a shoulder at around $2.38 \mathrm{eV}(520 \mathrm{~nm}$, Fig. 2a). The emission features remain largely unchanged with increasing concentration of GOQDs. The shoulder component around $2.35 \mathrm{eV}$ is, however, slightly enhanced while the main emission peak remains at $2.48 \mathrm{eV}$. A rearrangement of the PDV.Li vibronic transitions was previously observed in a PDV.K:PEO system as a function of PEO loading ${ }^{[11 b]}$, although with a concomitant blue-shift of both peaks that we do not observe here.

It is worth noting that the PDV.Li PL quantum efficiency (PLQE) of these solutions is quenched by only $\sim 5 \%$ with loading of GOQD up to $20 \%$ (see Supporting Information for the PLQE as a function of GOQD concentration, Table S1). Similarly we do not observe significant changes in the temporal decay of the luminescence of PDV.Li, in the presence of GOQDs, nor a progressive effect as a function of loading (see Fig. S2). This is surprising because GO has been reported to be an efficient PL quencher ${ }^{[5,20]}$ due to charge/energy transfer. While some quenching of the PL appears to occur in our system, presumably by the negatively 
charged surface of GOQDs, this is minor, and does not compromise the overall efficiency of the active material. The behaviour described above is also markedly different with respect to those observed for the conjugated polyelectrolytes reported (PDV.Li and rotaxinated derivatives) for which either rotaxination, addition of PEO, polyviny-alcohol (PVA), ${ }^{[21]}$ or substitution with larger cations induce large increases of the PLQE, blue-shifts, and changes in the dynamics temporal decay (e.g. in the weights of excitons vs. aggregate luminescence). ${ }^{[9 b, 11-12]}$

We also investigated the PL of thin films of GOQDs, PDV.Li and relative blends in different weight ratios, as shown in Fig. 2d. The spectra of the neat PDV.Li films and that of the composite with $0.5 \%$ GOQDs loading are essentially identical, whereas higher loading with GOQDs induces an overall narrowing of the emission, accompanied by a minor blue-shift of the peak.
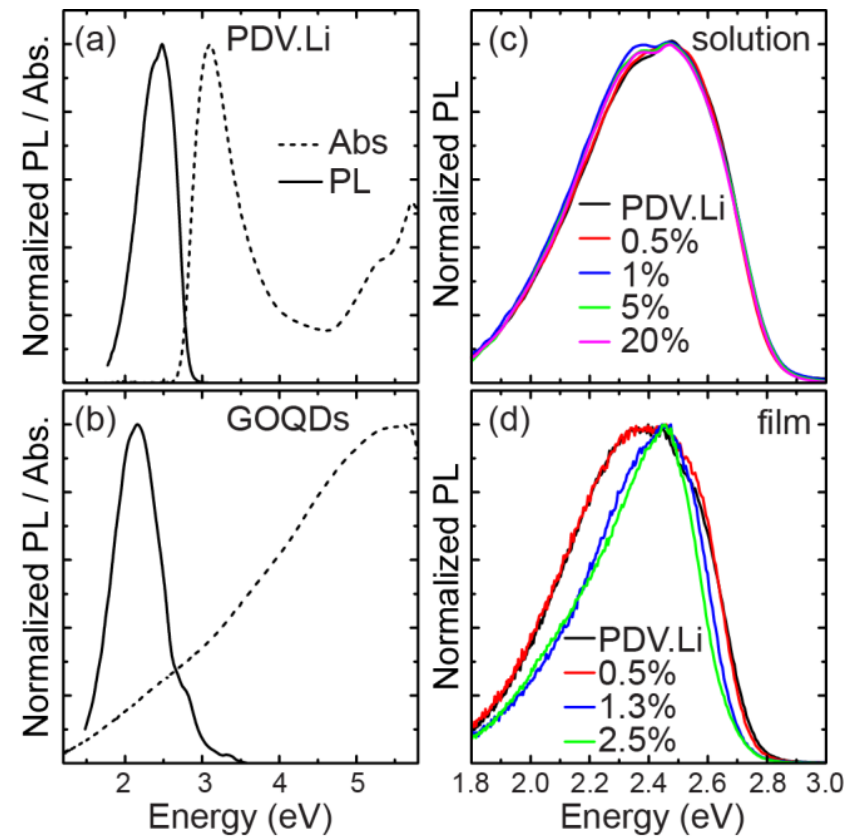

Figure 2 Optical absorption and PL spectra of neat (a) PDV.Li and (b) GOQDs in solution. PL spectra were collected using an $\mathrm{HeCd}$ laser $\left(\lambda_{\mathrm{ex}}=325 \mathrm{~nm}\right)$ as excitation source. The PL efficiency of the PDV.Li in solution is $23 \pm 2 \%$ whereas that of the GOQDs is less than $1 \%$. (c) Normalized PL spectra of PDV.Li:GOQDs blends in solutions in different ratios. (d) Normalized PL spectra of PDV.Li:GOQDs in films with different GOQDs loadings. The PL spectra in (c) and (d) were collected using a pulsed laser diode $\left(\lambda_{\mathrm{ex}}=371 \mathrm{~nm}\right)$ as excitation source.

Such a blue shift is most likely attributed to reduced aggregation of PDV.Li, similar to the case of rotaxination or encapsulation by PEO or amylose. ${ }^{[9 b, 22]}$ In this case, however, the PLQE does not show any noticeable enhancement (see Table S2). A possible explanation for the blue-shift is that positively charged lithium ions 
mediate a coulombic interaction between the negatively charged GOQDs and the negatively charged anionic polymeric cores, thereby hindering aggregation. Lack of strong PLQE modifications following the doping with GOQDs may be explained by the competition between two opposing effects: increase of the PLQE upon suppression of aggregation, and PL quenching induced by the GOQDs.

The surface morphology of the films containing different concentrations of GOQDs is shown in Fig. 3. The film roughness $\left(\mathrm{R}_{\mathrm{q}}\right)$ decreases from $2.11 \mathrm{~nm}$ (Fig. 3a) to $0.91 \mathrm{~nm}$ (Fig. 3d) with increasing concentration of GOQDs. This behaviour is somewhat similar to the change of polymer packing geometries induced via progressive cyclodextrin encapsulation in which the more rigid polymer backbones reduce the roughness of spin-coated films.
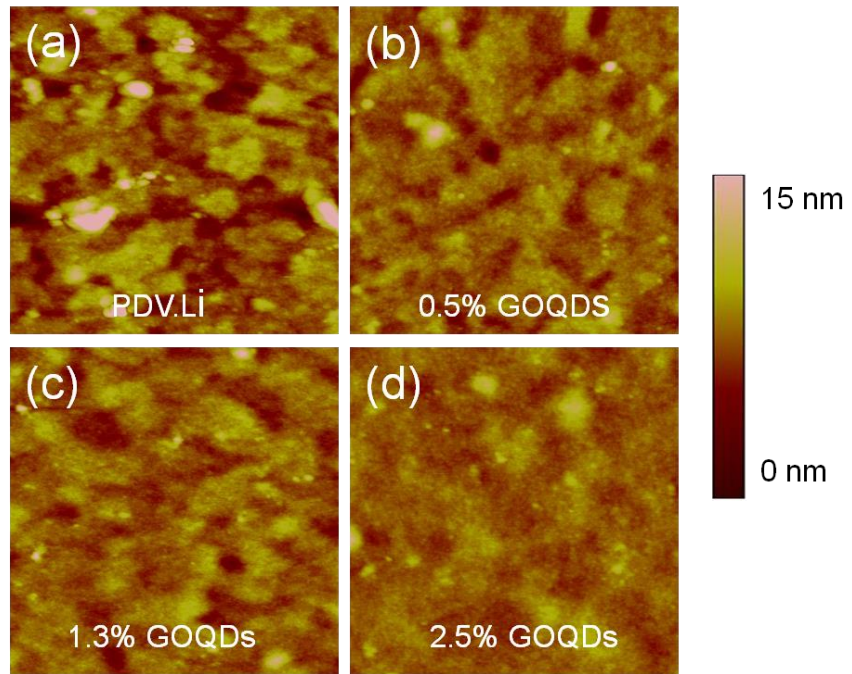

Figure $32 \times 2 \mu \mathrm{m}^{2}$ AFM images of PDV.Li-GOQDs composites on ITO substrates.

To obtain further insight into the properties of such composites we prepared and characterized the properties of LEDs having the PDV.Li:GOQDs composite film as the emissive layer. Fig. 4a shows the electroluminescence (EL) spectra for devices with up to $1.3 \%$ GOQD loading. It may be noted that we could not obtain accurate and reproducible results from devices at higher GOQD concentrations. As commonly observed, the EL spectra of the films are red-shifted (by 0.1 to $0.15 \mathrm{eV} \mathrm{nm}$ in this particular case, depending on the GOQDs loading) compared to the PL spectra. ${ }^{[23]}$ The EL spectra for the PDV.Li:GOQDs composites show enhanced peaks/shoulders at $2.08 \mathrm{eV}$ and $2.55 \mathrm{eV}$, respectively. 

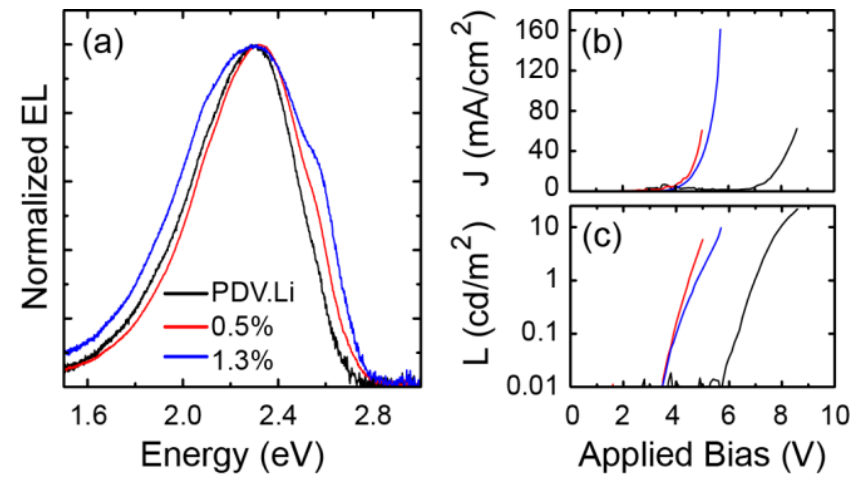

Figure 4 (a) Normalized EL of devices. (b) Current density and (c) luminance of devices with different loads of GOQDs with different loads of GOQDs

In figures $4 \mathrm{~b}$ and $\mathrm{c}$ we show current density and luminance against voltage $(J-L-V)$ characteristics. Interestingly, the device turn-on voltage (measured as the voltage for which the luminance exceeds $0.01 \mathrm{~cd} / \mathrm{m}^{2}$ ) reduces from $\sim 5.8 \mathrm{~V}$ for the neat polymer device, to $3.6 \mathrm{~V}$ for devices with $0.5 \%$ and $1.3 \%$ GOQDs loading. Consistently, the current density significantly increases for a fixed voltage as a function of the GOQDs loading, thus indicating either improved mobility in the active layer or reduced barriers to charge injection. The EQE of the blend devices (see Fig. S3, 0.5\%, 1.3\%) is lower compared with pure PDV.Li device due to the aforementioned increase in conductivity of the device, which is not followed by an increase in luminance. We consider this effect to be due to a worsening of the charge carrier balance rather than to additional quenching which the PL experiments above seem to exclude. Despite the low values, it must be noted that there is no EQE drop-off with current density and/or GOQDs loading.

In conclusion, GOQDs only marginally affect the light-emitting properties of PDV.Li in both solutions and films, inducing a PLQE quenching of ca. 5\% in solution followed by small modifications of the PL spectral shape in both cases. More importantly, the GOQDs greatly reduced the turn-on voltage of PDV.Li based LEDs either as a result of enhanced charge mobility in the active layer or reduced barriers to injection at one or both of the electrodes. We propose that this enhancement, combined with additional device optimisation strategies such as introduction of optimised charge transport or blocking layers, ${ }^{[24]}$ and electrode work function engineering, ${ }^{[25]}$ could be exploited to reduce the operational voltage of organic light- 
emitting diodes, thus also improving their operational lifetime, ${ }^{[26]}$ as a result of lower power dissipation on the actual devices.

\section{Experimental:}

GOQDs synthesis: The GOQDS were synthesized using GO and a modified Hummers method. ${ }^{[27]}$ After drying, a GO sheet was formed and then thermally reduced at $500{ }^{\circ} \mathrm{C}$ in a tube furnace under forming gas flow. GOQDs were obtained by ultrasonicating the $\mathrm{rGO}$ sheet in a mixture of concentrated $\mathrm{H}_{2} \mathrm{SO}_{4}$ and $\mathrm{HNO}_{3}$. This process leads to re-oxidation and cutting of rGO sheet into nanometer sized ensembles. The product was purified by repeated filtration with DI water. The obtained GOQDs water solution was sonicated for $30 \mathrm{~min}$ before being used.

PDV.Li:GOQDs solutions preparation: Optical studies were carried out on water solutions of PDV.Li $(0.05 \mathrm{mg} / \mathrm{ml})$. Different amount of GOQDs were added to the PDV.Li water solutions obtaining concentrations: $0.5 \%, 1 \%, 5 \%$ and $20 \%$ (by weight) thus maintaining the PDV.Li concentration constant at $0.05 \mathrm{mg} / \mathrm{ml}$. None of the solutions were filtered to minimize uncertainties in concentration caused by material loss. After adding GOQDs, the solutions were sonicated for 10 minutes to ensure good mixing of the two components.

PDV.Li:GOQDs films preparation: Two starting water solutions were prepared, namely: A and B. Solution A contained PDV.Li with a concentration of $20 \mathrm{mg} / \mathrm{ml}$. Solution B contained $20 \mathrm{mg} / \mathrm{ml}$ of PDV.Li (as in solution A) and $0.5 \mathrm{mg} / \mathrm{ml}$ of GOQDs. Solutions $\mathbf{A}$ and $\mathbf{B}$ were then mixed to prepare solutions with different ratios of GOQDs to PDV.Li, namely $0.5 \%, 0.8 \%$ and $1.3 \%$, maintaining the PDV.Li concentration constant. Therefore, the highest ratio of GOQDs to PDV.Li in a blend is $2.5 \%$ by weight (solution B). Films deposition was carried out by spin-coating to obtain uniform films with a suitable thickness $(\sim 100 \mathrm{~nm})$.

Optical absorption and photoluminescence measurements: UV-visible absorption spectra were obtained with an Agilent 8453 spectrophotometer. PL spectra of GOQDs and PDV.Li solutions were excited with a HeCd laser $(\lambda=325 \mathrm{~nm})$, and collected with an Andor Newton EMCCD camera coupled to an Andor SHAMROCK 163 spectrograph. Solutions PL quantum efficiencies (PLQE) were measured relative to a quinine sulfate dehydrate in $1.0 \mathrm{~N}$ sulfuric acid solution $(\approx$ 
10-5 M, PLQE $=54.6 \pm 5 \%$, NIST standard reference material SRM\# 936a, www.nist.gov/srm) using a time-correlated single photon counting (TCSPC) unit (Edinburgh instruments, F900, time response $\approx 150 \mathrm{ps}$ ) and a pulsed laser diode ( $\lambda$ ex $=371 \mathrm{~nm}$, pulse width $\approx 40 \mathrm{ps}$ ) as the excitation source. PL time decay measurements were performed in TCSPC unit with an excitation at $500 \mathrm{~nm}$ wavelength.

Light-emitting diodes fabrication: Indium tin oxide (ITO) substrates were ultrasonically cleaned in acetone and isopropanol, followed by $\mathrm{O}_{2}$ plasma treatment ${ }^{[28]}$ for 5 min before the PDV.Li:GOQDs water solutions were spin-coated on top of it. Finally, $50 \mathrm{~nm}$ calcium and $150 \mathrm{~nm}$ aluminium were thermally evaporated as top electrodes. The active layer was about $100 \mathrm{~nm}$ thick, (as measured by a Dektak surface profilometer). Electroluminescence (EL) spectra were measured with the same set-up used for PL measurements, while the current-light-voltage $(J-L-V)$ measurements were carried out using a Keithley 2400 sourcemeter to drive the device, and a calibrated silicon photodetector connected to a Keithley 2000 multimeter to detect the light.

\section{Supporting Information}

Supporting Information is available from the Wiley Online Library or from the author

\section{Acknowledgements}

We thank the EC - Marie Curie ITN-GENIUS (PITN-GA-2010-264694) RTNTHREADMILL (MRTN-CT-2006-036040) for funding support. P. Li thanks the Higher Education Funding Council for England (HEFCE) for the Overseas Research Scholarship (ORS), and China Scholarship Council (CSC). GE acknowledges the financial support from Centre for Advanced Structural Ceramics (CASC) at Imperial College London. GE also acknowledges the Royal Society for the Newton International Fellowship. 


\section{References}

[1] aA. K. Geim, K. S. Novoselov, Nat. Mater. 2007, 6, 183-191; bZ. H. Chen, Y. M. Lin, M. J. Rooks, P. Avouris, Physica E 2007, 40, 228-232; cS. Stankovich, D. A. Dikin, G. H. B. Dommett, K. M. Kohlhaas, E. J. Zimney, E. A. Stach, R. D. Piner, S. T. Nguyen, R. S. Ruoff, Nature 2006, 442, 282-286; dX. Wang, L. J. Zhi, K. Mullen, Nano Lett. 2008, 8, 323-327; eK. S. Kim, Y. Zhao, H. Jang, S. Y. Lee, J. M. Kim, J. H. Ahn, P. Kim, J. Y. Choi, B. H. Hong, Nature 2009, 457, 706-710; fS. Bae, H. Kim, Y. Lee, X. F. Xu, J. S. Park, Y. Zheng, J. Balakrishnan, T. Lei, H. R. Kim, Y. I. Song, Y. J. Kim, K. S. Kim, B. Ozyilmaz, J. H. Ahn, B. H. Hong, S. lijima, Nat. Nanotechnol. 2010, 5, 574-578; gF. Bonaccorso, Z. Sun, T. Hasan, A. C. Ferrari, Nat. Photon. 2010, 4, 611-622; hG. Eda, G. Fanchini, M. Chhowalla, Nat. Nanotechnol. 2008, 3, 270-274.

[2] aD. A. Dikin, S. Stankovich, E. J. Zimney, R. D. Piner, G. H. B. Dommett, G. Evmenenko, S. T. Nguyen, R. S. Ruoff, Nature 2007, 448, 457-460; bG. Eda, M. Chhowalla, Adv. Mater. 2010, 22, 2392-2415; cD. R. Dreyer, S. Park, C. W. Bielawski, R. S. Ruoff, Chem. Soc. Rev. 2010, 39, 228-240; dK. A. Mkhoyan, A. W. Contryman, J. Silcox, D. A. Stewart, G. Eda, C. Mattevi, S. Miller, M. Chhowalla, Nano Lett. 2009, 9, 1058-1063; eS. S. Li, K. H. Tu, C. C. Lin, C. W. Chen, M. Chhowalla, ACS Nano 2010, 4, 3169-3174; fK. P. Loh, Q. Bao, G. Eda, M. Chhowalla, Nat Chem 2010, 2, 1015-1024; gX. M. Sun, Z. Liu, K. Welsher, J. T. Robinson, A. Goodwin, S. Zaric, H. J. Dai, Nano Res. 2008, 1, 203-212.

[3] G. Eda, Y.-Y. Lin, C. Mattevi, H. Yamaguchi, H.-A. Chen, I. S. Chen, C.-W. Chen, M. Chhowalla, Adv. Mater. 2010, 22, 505-509.

[4] L. Tang, R. Ji, X. Cao, J. Lin, H. Jiang, X. Li, K. S. Teng, C. M. Luk, S. Zeng, J. Hao, S. P. Lau, ACS Nano 2012, 6, 5102-5110.

[5] H. Dong, W. Gao, F. Yan, H. Ji, H. Ju, Anal. Chem. 2010, 82, 5511-5517.

[6] aZ. F. Liu, Q. Liu, Y. Huang, Y. F. Ma, S. G. Yin, X. Y. Zhang, W. Sun, Y. S. Chen, Adv. Mater. 2008, 20, 3924; bY. Li, Y. Hu, Y. Zhao, G. Shi, L. Deng, Y. Hou, L. Qu, Adv. Mater. 2011, 23, 776-780.

[7] J. Luo, L. J. Cote, V. C. Tung, A. T. L. Tan, P. E. Goins, J. Wu, J. Huang, J. Amer. Chem. Soc. 2010, 132, 17667-17669.

[8] P. J. Zomer, S. P. Dash, N. Tombros, B. J. van Wees, Appl .Phys. Lett. 2011, 99, 232104-232103.

[9] aF. Cacialli, J. S. Wilson, J. J. Michels, C. Daniel, C. Silva, R. H. Friend, N. Severin, P. Samori, J. P. Rabe, M. J. O'Connell, P. N. Taylor, H. L. Anderson, Nat Mater 2002, 1, 160-164; bS. Brovelli, G. Latini, M. J. Frampton, S. O. McDonnell, F. E. Oddy, O. Fenwick, H. L. Anderson, F. Cacialli, Nano Lett. 2008, 8, 4546-4551; cA. Petrozza, S. Brovelli, J. J. Michels, H. L. Anderson, R. H. Friend, C. Silva, F. Cacialli, Adv. Mater. 2008, 20, 3218-3223.

[10] J. S. Wilson, M. J. Frampton, J. J. Michels, L. Sardone, G. Marletta, R. H. Friend, P. Samorì, H. L. Anderson, F. Cacialli, Adv. Mater. 2005, 17, 2659-2663.

[11] aG. Latini, L.-J. Parrott, S. Brovelli, M. J. Frampton, H. L. Anderson, F. Cacialli, Adv. Funct. Mater. 2008, 18, 2419-2427; bG. Latini, G. Winroth, S. Brovelli, S. O. McDonnell, H. L. Anderson, J. M. Mativetsky, P. Samorì, F. Cacialli, J. Appl. Phys. 2010, 107, 124509-124517.

[12] S. Brovelli, T. Virgili, M. M. Mroz, G. Sforazzini, A. Paleari, H. L. Anderson, G. Lanzani, F. Cacialli, Adv. Mater. 2010, 22, 3690-3694.

[13] aS. Brovelli, F. Meinardi, G. Winroth, O. Fenwick, G. Sforazzini, M. J. Frampton, L. Zalewski, J. A. Levitt, F. Marinello, P. Schiavuta, K. Suhling, H. L. Anderson, F. Cacialli, Adv. Funct. Mater. 2010, 20, 272-280; bS. Brovelli, G. Sforazzini, M. Serri, G. Winroth, K. Suzuki, F. Meinardi, H. L. Anderson, F. Cacialli, Adv. Funct. Mater. 2012, 22, 4284-4291. 
[14] C. R. Dean, A. F. Young, I. Meric, C. Lee, L. Wang, S. S., K. Watanabe, T. Taniguchi, P. Kim, K. L. Shepard, J. Hone, Nat. Nanotechnol. 2010, 5, 722-726.

[15] J. Kim, L. J. Cote, F. Kim, J. Huang, Journal of the American Chemical Society 2009, 132, 260-267.

[16] Z. Luo, Y. Lu, L. A. Somers, A. T. C. Johnson, J. Amer. Chem. Soc. 2009, 131, 898899.

[17] aD. Pan, J. Zhang, Z. Li, M. Wu, Adv. Mater. 2010, 22, 734-738; bJ. Peng, W. Gao, B. K. Gupta, Z. Liu, R. Romero-Aburto, L. Ge, L. Song, L. B. Alemany, X. Zhan, G. Gao, S. A. Vithayathil, B. A. Kaipparettu, A. A. Marti, T. Hayashi, J.-J. Zhu, P. M. Ajayan, Nano Lett. 2012, 12, 844-849.

[18] S. Zhu, J. Zhang, C. Qiao, S. Tang, Y. Li, W. Yuan, B. Li, L. Tian, F. Liu, R. Hu, H. Gao, H. Wei, H. Zhang, H. Sun, B. Yang, Chem. Commun. 2011, 47, 6858-6860.

[19] C.-T. Chien, S.-S. Li, W.-J. Lai, Y.-C. Yeh, H.-A. Chen, I. S. Chen, L.-C. Chen, K.-H. Chen, T. Nemoto, S. Isoda, M. Chen, T. Fujita, G. Eda, H. Yamaguchi, M. Chhowalla, C.-W. Chen, Angew. Chem. Int. Ed 2012, 51, 6662-6666.

[20] aE. Treossi, M. Melucci, A. Liscio, M. Gazzano, P. Samorì, V. Palermo, J. Am. Chem. Soc. 2009, 131, 15576-15577; bS. Li, A. N. Aphale, I. G. Macwan, P. K. Patra, W. G. Gonzalez, J. Miksovska, R. M. Leblanc, ACS Appl. Mater. 2012, 4, 7069-7075.

[21] F. Di Stasio, P. Korniychuk, S. Brovelli, P. Uznanski, S. O. McDonnell, G. Winroth, H. L. Anderson, A. Tracz, F. Cacialli, Adv. Mater. 2011, 23, 1855-1859.

[22] M. J. Frampton, T. D. W. Claridge, G. Latini, S. Brovelli, F. Cacialli, H. L. Anderson, Chem. Commun. 2008, 2797-2799.

[23] O. Fenwick, S. Fusco, T. N. Baig, F. Di Stasio, T. T. Steckler, P. Henriksson, C. Fléchon, M. R. Andersson, F. Cacialli, APL Materials 2013, 1, -.

[24] aG. M. Lazzerini, F. Di Stasio, C. Flechon, D. J. Caruana, F. Cacialli, Appl .Phys. Lett. 2011, 99; bG. Winroth, G. Latini, D. Credgington, L.-Y. Wong, L.-L. Chua, P. K. H. Ho, F. Cacialli, Appl .Phys. Lett. 2008, 92.

[25] aN. Crivillers, S. Osella, C. Van Dyck, G. M. Lazzerini, D. Cornil, A. Liscio, F. Di Stasio, S. Mian, O. Fenwick, F. Reinders, M. Neuburger, E. Treossi, M. Mayor, V. Palermo, F. Cacialli, J. Cornil, P. Samori, Adv. Mater. 2013, 25, 432-436; bG. M. Lazzerini, S. Mian, F. Di Stasio, A. M. Masillamani, N. Crivillers, F. Reinders, M. Mayor, P. Samori, F. Cacialli, Applied Physics Letters 2012, 101; cN. Crivillers, A. Liscio, F. Di Stasio, C. Van Dyck, S. Osella, D. Cornil, S. Mian, G. M. Lazzerini, O. Fenwick, E. Orgiu, F. Reinders, S. Braun, M. Fahlman, M. Mayor, J. Cornil, V. Palermo, F. Cacialli, P. Samori, Phys. Chem. Chem. Phys. 2011, 13, 14302-14310.

[26] aF. Cacialli, X. C. Li, R. H. Friend, S. C. Moratti, A. B. Holmes, Synthetic Metals 1995, 75, 161-168; bJ. S. Kim, F. Cacialli, R. H. Friend, R. Daik, W. J. Feast, Synth. Met. 1999, 102, 1065-1066; cG. Winroth, S. Brovelli, R. Daik, W. J. Feast, F. Cacialli, Organic Electronics 2010, 11, 1445-1448.

[27] aM. Hirata, T. Gotou, S. Horiuchi, M. Fujiwara, M. Ohba, Carbon 2004, 42, 29292937; bG. Eda, G. Fanchini, M. Chhowalla, Nat. Nanotechnol. 2008, 3, 270-274.

[28] T. M. Brown, F. Cacialli, J. Polym. Sci., Part B: Polym. Phys. 2003, 41, 2649-2664. 